\title{
Comparison of choroidal thickness in eyes of diabetic patients with eyes of healthy individuals using optical coherence tomography in a tertiary care hospital
}

\author{
Hafsa Hassan ${ }^{1}$, Alyscia Cheema², \\ Muhammad Ali Tahir ${ }^{3}$, Hina Nasreen Nawaz ${ }^{4}$
}

\begin{abstract}
Objectives: To compare the choroidal thickness in eyes of diabetic patients with eyes of age matched controls using optical coherence tomography in a tertiary care hospital.

Methods: This Cross sectional study was conducted at the Department of Ophthalmology, Jinnah Postgraduate Medical Centre Karachi, for six months from $13^{\text {th }}$ January 2020 to $13^{\text {th }}$ July 2020 . The study group comprised of 44 patients with 88 eyes. Patients who fulfill the inclusion criteria that is age ranging from 35 to 80years, either gender, known case of diabetes mellitus and having any type of diabetic retinopathy (HbA1C >7), non-diabetic healthy individuals ( $\mathrm{HbA1C}<7)$ and those giving informed consent were included in the study. However, patients having active ocular infections, history of myocardial infarction, stroke, uveitis, any ocular surgery, lasers, intravitreal injections, poor fundus view and not giving consent were excluded. A pre-designed proforma was filled. A baseline ocular examination was performed and choroidal thickness was assessed from retinal pigment epithelium to choroid sclera junction in diabetic and healthy participants of the study group using high resolution Swept source OCT (DRI-OCT-2 Triton; Topcon).

Results: The average age of the patients was $39.41 \pm 15.95$ years. According to our study mean central subfoveal choroidal thickness in diabetic eyes was $268.5 \pm 66.22(95 \% \mathrm{Cl} 240-297)$ and in non-diabetic healthy participants it was $339.3 \pm 71.49(95 \% \mathrm{Cl} 308-369)$ with a p-value of 0.001 . However, average choroidal thickness was $261.8 \pm 61.93(95 \% \mathrm{Cl} 235-288)$ and $336.0 \pm 74.35(95 \% \mathrm{Cl} 304-367)$ in diabetic and non-diabetic healthy population with a $p$-value of 0.001 . Choroidal thickness comparison between gender in diabetic and non-diabetic population also showed similar trend.

Conclusion: In this study, mean central choroidal thickness as well as average choroidal thickness was significantly reduced in eyes having diabetic retinopathy as compared to participants with non-diabetic healthy eyes. These findings indicate that changes in choroid may be a probable route in the pathogenesis of diabetic retinopathy.
\end{abstract}

KEYWORDS: Choroidal thickness, Swept source optical coherence tomography, Diabetic retinopathy.

How to cite this:

doi: https://doi.org/10.12669/pjms.38.1.4443

Hassan H, Cheema A, Tahir MA, Nawaz HN. Comparison of choroidal thickness in eyes of diabetic patients with eyes of healthy individuals using optical coherence tomography in a tertiary care hospital. Pak J Med Sci. 2022;38(1):254-260. doi: https://doi.org/10.12669/pjms.38.1.4443

This is an Open Access article distributed under the terms of the Creative Commons Attribution License (http://creativecommons.org/licenses/by/3.0), which permits unrestricted use, distribution, and reproduction in any medium, provided the original work is properly cited.

Correspondence:

Muhammad Ali Tahir, MBBS, FCPS (Ophthalmology),

FCPS (Vitreoretina),

Consultant Retinal Surgeon,

Jinnah Post Graduate Medical Centre,

Karachi, Pakistan.

E mail: ali.tahir81@gmail.com

* Received for Publication:

* Revision Received:

* Revision Accepted:
March 19, 2021

September 2, 2021

September 18, 2021

\section{INTRODUCTION}

The rise of diabetic population in the world has led to a number of problems. Diabetic retinopathy (DR) damages vision by causing abnormalities in retinal microvasculature and capillaries. ${ }^{1}$ The disease is described by development of micro aneurysms, perfusion abnormalities of capillaries, 
and ischemic retina, eventually heading to neo vessels formation and macular edema, which can disturb visual function. ${ }^{2,3}$ Clinically significant macular edema is edema or hard exudates within 500 micrometers of the centre of macula, retinal edema one disc diameter or larger, any part of which is within one disc diameter of center of macula. The outer third of the retina gets oxygen and nutrients from choroid. The three vascular layers of choroid are chorio capillaries layer, Sattler layer and Haller layer.,4 Since there are many diseases of posterior segment which also involve choroid any assessment of morphological features and its vasculature in different chorioretinal diseases may be of clinical significance. Hemodynamic abnormalities, Changes in retinal vessels integrity, break down of blood retina barrier mark the development of diabetic eye disease. ${ }^{5}$ Clinicians and researchers believe that in addition to retinal changes choroidal vasculopathy might also play some role in the development of diabetic retinopathy.

Numerous choroidal malfunctions including blockades of the choriocapillaries, disintegration of vessels, aneurysms of vessels of choroid, and choroidal neovascularization have been testified in diabetic eyes. ${ }^{6}$ Good-quality with higherresolution cross-sectional macula imaging is possible with Spectral-domain optical coherence tomography (OCT). Choroidal thickness is different in diabetic and non-diabetic eyes as per previous studies. Adhi et $\mathrm{al}^{2}$ observed alteration in morphology of choroid while comparing mild to moderate NPDR patients to normal population and showed that mean choroidal thickness was $276.4 \pm 13.4$ micrometers in normal eyes while $211.6 \pm 17$ um in diabetic eyes. The mid of choroid is attenuated in case of diabetic retinopathy. Whereas, Lee et $\mathrm{al}^{5}$ showed that the mean central choroid was $229.1 \pm 16.8 \mu \mathrm{m}$ in normal eye compared to $219.7 \pm 30.4 \mu \mathrm{m}$ in diabetic eye. Similarly, Gerendas et $\mathrm{al}^{7}$ also concluded that choroidal thickness was decreased in patients with diabetes if DME was present. Since prevalence of diabetes is increasing it is imperative to prevent its ophthalmological complications for individual to live a normal life. We can prevent irreversible blindness due to diabetes by looking at choroidal thickness beforehand and patients can be counseled and treated before irreversible blindness occur. We are therefore comparing the choroidal thickness of diabetic and non-diabetic eyes using swept source OCT.

\section{METHODS}

The study was conducted at Department of Ophthalmology, Jinnah Post-Graduate Medical Centre, Karachi from $13^{\text {th }}$ January 2020 to $13^{\text {th }}$ July 2020. Sample size calculation was done by WHO sample size calculator using the following assumptions. ${ }^{8}$ Confidence Interval (1- $\left.\alpha\right)=$ $95 \%$. Margin of error (d) 0.03 Taking choroidal thickness $259.1 \pm 13.1$ um. Sample size of 88 eyes of 44 patients was calculated. Out of which 44 eyes were of diabetic patients having diabetic retinopathy $(\mathrm{HbA1c}>7)$ and 44 eyes were of nondiabetic healthy individuals age matched control group (HbA1c <7). Approval of the study was done by institutional review board and ethical committee of Jinnah Post Graduate Medical Centre (Ref: No.F.2-81/2020=GENL/S374/PJMC, Dated: 03-02-2020). Patients having either gender, age between 35 to 80 years, any type of diabetic retinopathy, duration of diabetes (at least greater than one year), non-diabetic healthy individuals and those who gave consent for inclusion in the study and for OCT were included in the study. However patients having active ocular infection, uveitis, uncontrolled hypertension, pregnancy, any ocular surgery, lasers or intravitreal injections and those who did not give consent were excluded from the study. A pre-designed proforma was filled. A baseline ocular examination was performed and choroidal thickness was assessed using swept source OCT (SS-OCT) (DRI-OCT-2 Triton; Topcon). It was measured from retinal pigment epithelium to choroid scleral junction. Choroidal thickness was measured in central subfoveal region, 500um temporal to central region and 500um nasal to central sub foveal region. Central subfoveal and average thickness of three regions were compared between diabetic and non-diabetic healthy individuals of the study group. SPSS version 21 was used for data entry and analysis. Mean (SD) was computed for Age, duration of diabetes mellitus and choroidal thickness. Frequencies and percentages were calculated for categorical variables like gender, diabetics and non-diabetics. Effect modifiers such as age, was assessed through stratification. Post stratification, Dependent/paired T-test was applied. P-value $\leq 0.05$ was considered significant.

\section{RESULTS}

Mean age of patients was $39.41 \pm 15.95$ years. Average duration of diabetes was $10.64 \pm 2.7$ years. 
Table-I: Mean comparison of the Choroidal thickness in eyes of diabetic patients with eyes of healthy individuals using optical coherence tomography (OCT) (n= 44 patients with 88 eyes).

\begin{tabular}{|c|c|c|c|c|c|}
\hline \multirow{2}{*}{ Choroidal Thickness } & \multicolumn{2}{|c|}{ Diabetic $(n=22)$} & \multicolumn{2}{|c|}{ Non diabetic $(n=22)$} & \multirow{2}{*}{$p$-valuc } \\
\hline & Mean \pm S.D & (95\% C.I) & Mean $\pm S . D$ & $95 \%$ C.I & \\
\hline Nasal & $253.5 \pm 62.23$ & $(227-280)$ & $330.4 \pm 74.54$ & $(298-362)$ & 0.001 \\
\hline Central & $268.5 \pm 66.22$ & $(240-297)$ & $339.3 \pm 71.49$ & $(308-369)$ & 0.001 \\
\hline Temporal & $263.5 \pm 61.00$ & $(237-289)$ & $388.7 \pm 79.01$ & $(304-372)$ & 0.001 \\
\hline Average choroidal thickness & $261.8 \pm 61.93$ & $(235-288)$ & $336.0 \pm 74.35$ & $(304-367)$ & 0.001 \\
\hline
\end{tabular}

For Male Cases ( $\mathrm{n}=28$ patients with 56 eyes).

Out of 88 eyes of 44 patients 32 eyes (36.6\%) were of female patients and 56 eyes $(63.64 \%)$ were of male patients. Mean central choroidal thickness in patients having diabetic retinopathy was $268.5 \pm$ 66.22 (95\% CI 240 - 297). However, it was $339.3 \pm$ 71.49 (95\% CI 308 - 369) in normal healthy subjects hence was significantly reduced in comparison to the normal healthy subjects with a p-value of 0.001 . Average choroidal thickness was calculated by taking mean of central, nasal and temporal thickness readings. Average choroidal thickness in patients having diabetic retinopathy was $261.8 \pm 61.93$ (95\% CI $235-288)$ in comparison to $336.0 \pm 74.35(95 \% \mathrm{CI}$ $304-367)$ in normal healthy subjects with a p-value of 0.001. Similarly, nasal and temporal thicknesses were also reduced significantly in diabetic population of the group in comparison to normal healthy population as shown in Table-I. Same trend was seen when stratification was done according to gender. Significant difference in central and average thicknesses was seen in male gender in diabetic and non-diabetic study population of group (Table-II). However in case of female gender although the difference was observed but it was not significant as shown in (Table-III). Similarly, nasal and temporal thicknesses were also reduced significantly in diabetic population of the group in comparison to normal healthy population as shown in Table-I. Same trend was seen when stratification was done according to gender. Significant difference in central and average thicknesses was seen in male gender in Diabetic and non diabetic study population of the group. (Table-II) however in case of female gender although the difference was observed but it was not significant as shown in (Table-III).

\section{DISCUSSION}

Choroid having normal structure and function is imperative for the functioning of retina. Unusual blood volume of choroid or reduced blood flow can cause malfunction of photoreceptors and their death as well. ${ }^{9}$

Number of studies was conducted previously investigating the effect of choroidal vessels in the development of diabetic retinopathy. It was speculated that almost similar changes take place in retinal and choroidal vessels and growth factors released by diabetic retina and choroid were also similar. It was assumed that affected choroidal vascular system in diabetic

Table-II: Mean Comparison of the Choroidal thickness in eyes of diabetic patients with eyes of healthy individuals using optical coherence tomography (OCT).

\begin{tabular}{|c|c|c|c|c|c|}
\hline \multirow{2}{*}{ Choroidal Thickness (Male) } & \multicolumn{2}{|c|}{ Diabetic $(n=12)$} & \multicolumn{2}{|c|}{ Non diabetic $(n=16)$} & \multirow{2}{*}{ p-value } \\
\hline & Mean \pm S.D & $(95 \%$ C.I) & Mean \pm S.D & (95\% C.I) & \\
\hline Nasal & $236.5 \pm 61.48$ & $(201-272)$ & $333.3 \pm 84.95$ & $(290-375)$ & 0.003 \\
\hline Central & $253.6 \pm 69.40$ & $(213-293)$ & $345.0 \pm 80.38$ & $(304-385)$ & 0.004 \\
\hline Temporal & $255.4 \pm 63.22$ & $(218-291)$ & $343.4 \pm 89.51$ & $(298-388)$ & 0.007 \\
\hline Average choroidal thickness & $248.5 \pm 63.95$ & $(211-285)$ & $340.58 \pm 84.27$ & $(298-382)$ & 0.001 \\
\hline
\end{tabular}

For Female Cases ( $\mathrm{n}=16$ patients). 
Hafsa Hassan et al.

Table-III: Mean Comparison of the Choroidal thickness in eyes of diabetic patients with eyes of healthy individuals using optical coherence tomography (OCT).

\begin{tabular}{|c|c|c|c|c|c|}
\hline \multirow{2}{*}{ Choroidal Thickness (Female) } & \multicolumn{2}{|c|}{ Diabetic $(n=10)$} & \multicolumn{2}{|c|}{ Non diabetic $(n=6)$} & \multirow{2}{*}{ p-value } \\
\hline & Mean \pm S.D & (95\% C.I) & Mean \pm S.D & $(95 \%$ C.I) & \\
\hline Nasal & $273.9 \pm 59.73$ & $(236-311)$ & $322.8 \pm 39.81$ & $(290-355)$ & 0.098 \\
\hline Central & $286.4 \pm 60.73$ & $(248-324)$ & $323.9 \pm 41.18$ & $(290-357)$ & 0.205 \\
\hline Temporal & $273.1 \pm 60.05$ & $(235-311)$ & $324.6 \pm 43.30$ & $(289-360)$ & 0.089 \\
\hline Average choroidal thickness & $277.8 \pm 58.56$ & $(240-314)$ & $323.8 \pm 40.88$ & $(290-357)$ & 0.115 \\
\hline
\end{tabular}

No significant difference were observed $\mathrm{p}>0.05$.

patients may be associated in the development of diabetic retinopathy. ${ }^{10}$ SD-OCT and so does swept source OCT has helped in evaluation of cross sectional structure of choroid. ${ }^{11}$ and choroid scleral interface. ${ }^{12}$ Studies evaluating the blood flow in sub foveal choroidal region using Doppler flowmetry reveal decline in volume and blood flow in patients suffering from moderate to severe non proliferative diabetic retinopathy (NPDR) and proliferative diabetic retinopathy (PDR). ${ }^{13}$ Reduction in blood flow was more marked in PDR. ${ }^{14}$

To understand Diabetic eye disease in a better way it is important to have knowledge of choroidal damage. It is only recently that appropriate visualization of choroid has been possible using OCT in spite of difficulties like posterior location and existence of retinal pigment epithelium. ${ }^{15,16}$

In this study the average age of the patients was $39.41 \pm 15.95$ years. $63.64 \%$ were male patients and $36.36 \%$ were female. In El Ghonemy et al study ${ }^{17}$ the mean age of the control group and the mean age of the diabetic patients were $52.88 \pm 12.9$ and $56.95 \pm 8.3$ years. In Wang et al study ${ }^{18}$ among them,58.28\% patients were female, the average age was $64.5 \pm 7.8$ years, the average duration of diabetes was $8.9 \pm 7.1$ years.

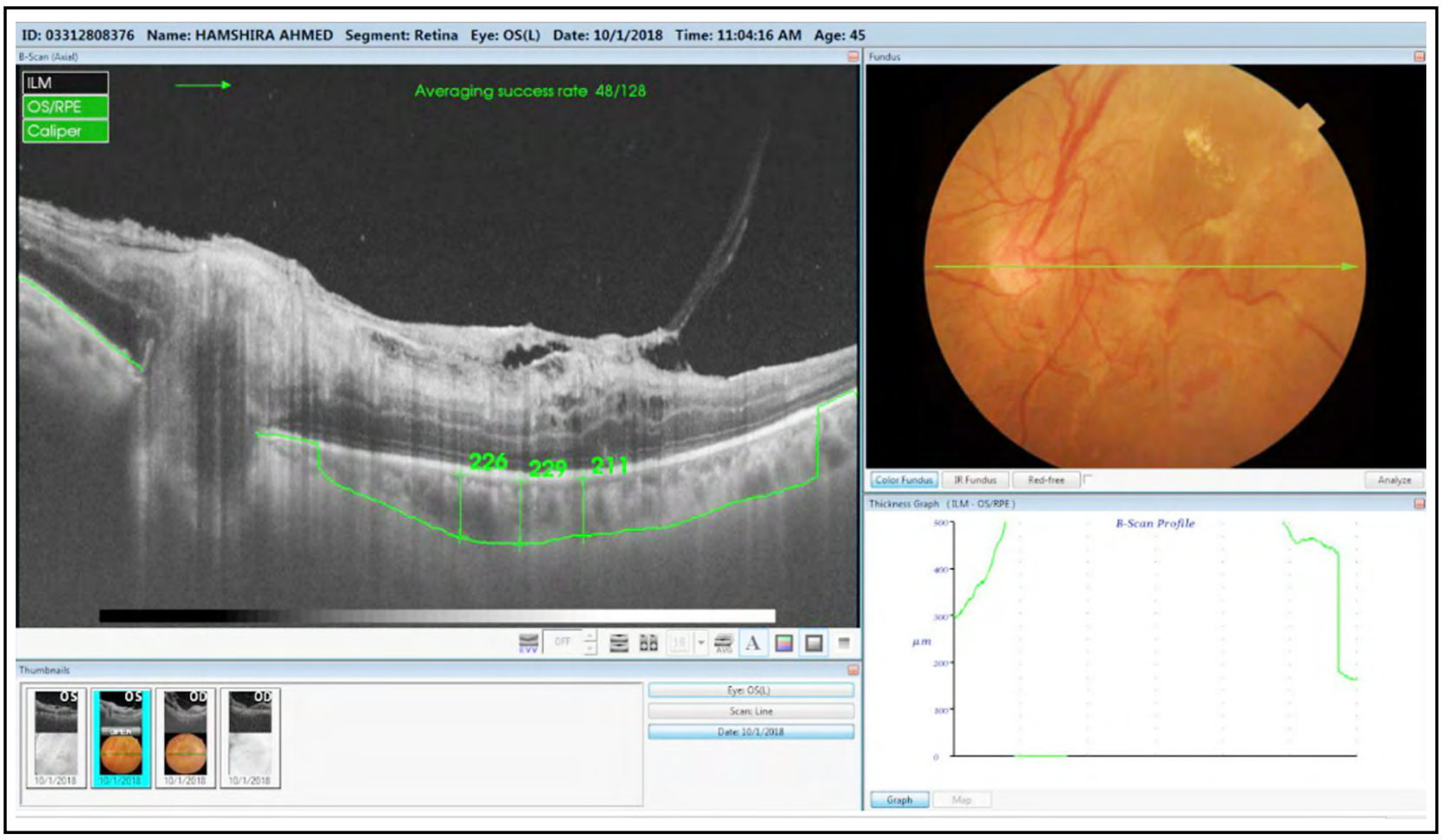

Fig.1: OCT image of a diabetic patient having PDR with DME illustrating choroidal thickness. 


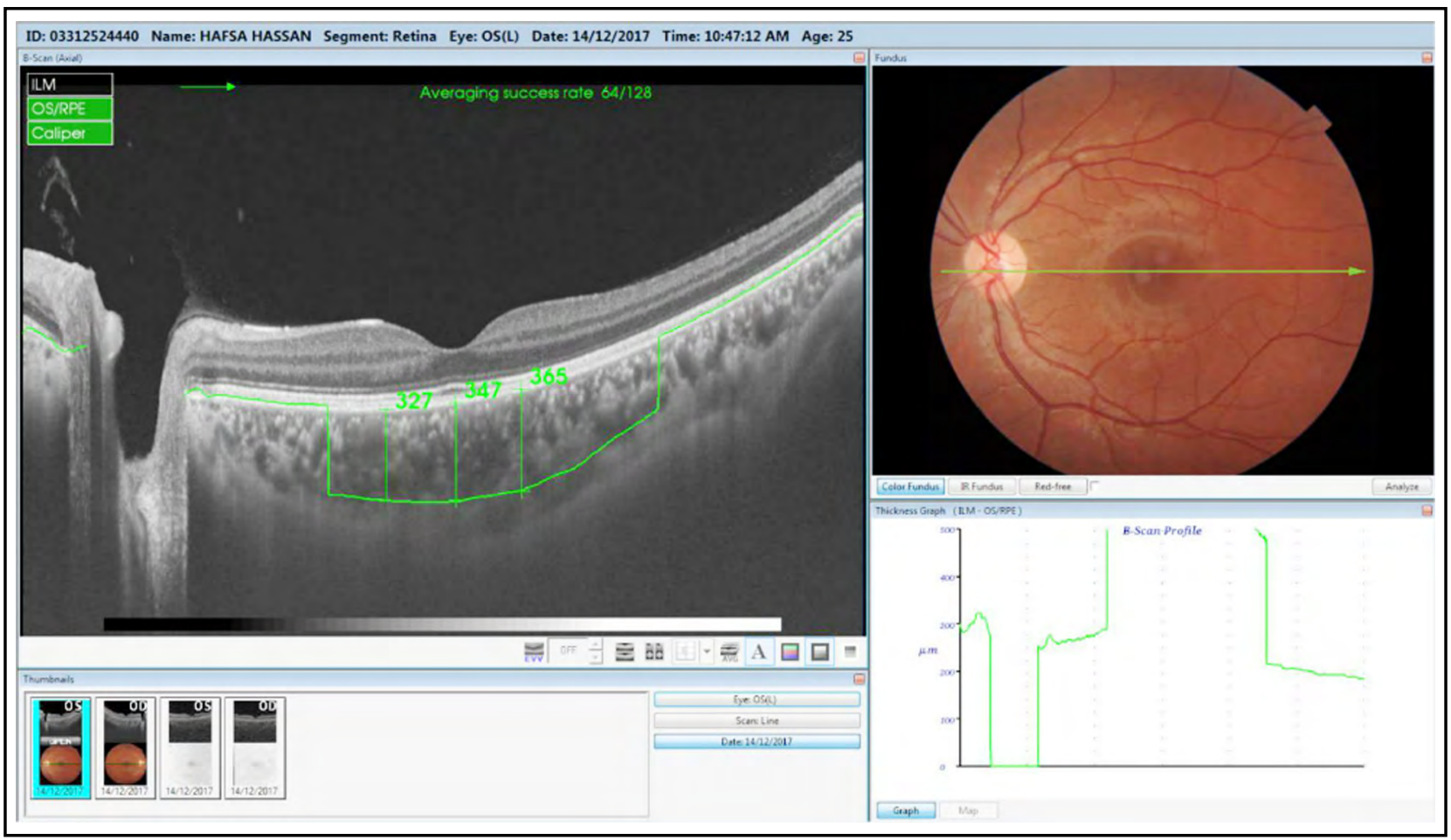

Fig.2: OCT image of a non diabetic healthy individual in our study group.

The difference in average age in our study group may be attributed to the patients who are coming from low socio economic status, poor control of diabetes and compliance to the treatment and follow up are also not good. Low percentage of females in our study group in comparison to Wang et $\mathrm{al}^{18}$ may be because of cultural values and females may not be reporting enough in comparison to males.

In our study mean central sub foveal choroidal thickness in normal healthy subjects was 339.3 \pm 71.49 um (CI 95\% 308-369) where as In El Ghonemy et al study ${ }^{17}$ mean subfoveal CT was $354.52 \pm 96.51 \mu \mathrm{m}$ in the control group $269.4 \pm 64.3 \mu \mathrm{m}$ in the mild NPDR subgroup, $240.7 \pm 67 \mu \mathrm{m}$ in the moderate NPDR subgroup, $227.7 \pm 47.9 \mu \mathrm{m}$ in the severe NPDR subgroup, $220.3 \pm 58.1 \mu \mathrm{m}$ in the PDR subgroup, and $224.4 \pm 55.3 \mu \mathrm{m}$ in the DME group. In our study mean central subfoveal choroidal thickness in eyes having diabetic retinopathy was $268.5 \pm 66.22$ (CI 95\% $240-297$ ). This value is very close to El Ghonemy et al mild and moderate NPDR sub groups. ${ }^{17}$

In Wang et al study ${ }^{18}$ the average CT was $189.2 \pm 72.6 \mu \mathrm{m}$ for all the participants, $187.6 \pm$ $72.5 \mu \mathrm{m}$ for patients without DR, and $195.4 \pm 72.9$ $\mu \mathrm{m}$ for DR patients $(\mathrm{P}=0.115)$. However, in our study average choroidal thickness in non-diabetic healthy sub group was $336.0 \pm 74.35$ (CI $95 \% 304$ - 367) and in diabetic sub group it was $261.8 \pm$ 61.93 (CI 95\% 235 - 288) with a p-value of 0.001 . In Wang et al study among measurements in nine sub regions, the CT showed a trend toward higher values in DR patients, but only CT in outer nasal region $(\mathrm{P}=0.015)$ and outer inferior region $(P=0.029)$ achieved statistical significance. In contrary to Wang et al study average choroidal and central subfoveal choroidal thicknesses in our study were not only higher in both diabetic and non-diabetic sub groups which may be attributed to different OCT machine used and different geographic location of people but the difference in choroidal thicknesses with in our study group were also significant with $p$ value of 0.001 . Mean central choroid was significantly low in diabetic eye as compared patients with nondiabetic eyes in this study. Whereas, Lee et $\mathrm{al}^{5}$ showed that the mean central choroid was 229.1 \pm $16.8 \mu \mathrm{m}$ in normal eye compared to $219.7 \pm 30.4$ $\mu \mathrm{m}$ in diabetic eye. Similarly, Gerendas et $\mathrm{al}^{7}$ also reported that choroidal thickness was reduced in patients with diabetes if DME was present. Studies done previously show that choroidal thickness varies in diabetic and non- diabetic eyes. Adhi et $\mathrm{al}^{2}$ showed that choroidal morphological features are altered in patients with moderate to 
severe diabetic retinopathy with mean chordial thickness of $276.4 \pm 13.4 \mu \mathrm{m}$ in normal eye while $211.6 \pm 17.0 \mu \mathrm{m}$ in diabetic eyes. Studies done previously show different results on association of choroidal thickness with severity of DR. Many authors concluded that choroidal thickness in DR patients is reduced. For example, Lains et al. ${ }^{19}$ demonstrated that $\mathrm{CT}$ in their proliferative DR group was thinner compared with controls. Horváth et al. ${ }^{20}$ and Ambiya et $\mathrm{al}^{21}$ revealed that decreasing CT correlated with the severity of DR. However, other studies reported thickening of choroid or no change with presence of DR. For example, Tavares et al. ${ }^{22}$ reported a thickening of choroid in diabetic patients without DR. The population-based Beijing Eye Study found that the DM was independently associated with a thicker choroid while the DR was not related to the choroidal thickness. ${ }^{23} \mathrm{DM}$ may act as an independent factor leading to choroid thickening and subsequent DR progression may lead to the reduction of $\mathrm{CT}$, which may appear as a thicker choroid at the initial stage of DR and thinning with DR progression. The choroid provides the outer layer of retina and the retinal pigment epithelium with oxygen and nutrients. Hence CT may give an idea about how much active retina and choroid are metabolically. The mechanism of CT alterations in DR remains unclear. Diabetic choroidopathy may result in RPE dysfunction, and affect vascular permeability. ${ }^{24}$ The increased choriocapillaries permeability leads to choroidal thickening. Second, the over expression of cytokines activated by inflammation, oxidative stress, angiogenesis in early DR may contribute to the thickening of the choroidal layer, such as monocyte chemotactic protein-1, platelet-derived growth factor, VEGF, insulin-like growth factor 1 , pigment epithelium-derived factor, and cxc motif chemokine ligand. ${ }^{25}$ It was reported that these cytokines were significantly associated with choroidal thickening. ${ }^{26,27}$ Third, the choroid accounts for $85 \%$ of ocular blood flow, autonomic nervous system was considered to be important for autoregulation of choroidal blood flow. In mild NPDR, sympathetic innervation increases choroidal circulation as a result of which choroidal thickness is increased. Savage et al. reported that the pulsatile ocular blood flow increased in DR eyes compared with controls using a computerized pneumotonometer. However, hypoxia plays a dominant role with the DR progression to late stage. ${ }^{28}$
Thinning of the choroid suggests a decrease in blood flow, and thus thinning of the choroid may be associated with hypoxia in the retinal tissue. However, further experimental studies are needed to determine whether choroidal thinning was primary or secondary to retinal ischemia. ${ }^{28}$

Limitation of the study: It was that average and central choroidal thicknesses among diabetic population were not compared according to the severity of Diabetic retinopathy like in sub groups of mild NPDR, moderate NPDR, severe NPDR, PDR and in advanced diabetic eye diseases to had an idea of decrease in choroidal thickness with increase in severity of diabetic retinopathy and severity of ischemia. More studies with greater sample size are required to find out the association between severity of diabetic retinopathy and degree of ischemia with reduction in choroidal thickness.

\section{CONCLUSION}

In this study mean central choroidal thickness was significantly low in diabetic eyes in comparison to patients with non-diabetic eyes. These findings lead to a point of view that change in choroidal thickness may be a route in the development of DR. More studies with greater sample size are required to find out the mechanism behind our findings.

\section{Grant support and financial disclosures: None.}

\section{REFERENCES}

1. Kim JT, Lee DH, Joe SG, Kim JG, Yoo YH. Changes in choroidal thickness in relation to the severity of retinopathy and macular edema in type 2 diabetic patients. Invest Ophthalmol Vis Sci. 2013;54(5):3378-3784

2. Adhi M, Brewer E, Waheed NK, Duker JS. Analysis of morphological features and vascular layers of choroid in diabetic retinopathy using spectral-domain optical coherence tomography. JAMA Ophthalmol. 2013;131(10):1267-1274.

3. Antonetti DA, Klein R, Gardner TW. Diabetic retinopathy. N Engl J Med. 2012;366(13):1227-1239.

4. KurJ, Newman EA, Chan-Ling T. Cellular and physiological mechanisms underlying blood flow regulation in the retina and choroid in health and disease. Prog Retin Eye Res. 2012;31(5):377-406

5. Lee HK, Lim JW, Shin MC. Comparison of choroidal thickness in patients with diabetes by spectral-domain optical coherence tomography. Korean J Ophthalmol. 2013;27(6):433-439.

6. Weinberger D, Kramer M, Priel E. Indocyanine green angiographic findings in nonproliferative diabetic retinopathy. Am J Ophthalmol. 1998;126:238-247. 
7. Gerendas BS, Waldstein SM, Simader C, DeakG, Hajnajeeb $\mathrm{B}$, Zhang Let al. Three-dimensional automated choroidal volume assessment on standard spectral-domain optical coherence tomography and correlation with the level of diabetic macular edema. Am J Ophthalmol. 2014;158(5):1039-1048.

8. Regatieri CV, Branchini L, Carmody J, Fujimoto JG, Duker JS. Choroidal thickness in patients with diabetic retinopathy analyzed by spectral-domain optical coherence tomography. Retina. 2012;32(3):563-568.

9. Cao J, Mc Leod S, Merges CA, Lutty GA. Chorio capillaris degeneration and related pathologic changes in human diabetic eyes. Arch Ophthalmol. 1998;116:589-597.

10. MacGregor LC, Rosecan LR, Laties AM, Matschinsky FM. Altered retinal metabolism in diabetes. I. Microanalysis of lipid, glucose, sorbitol, and myoinositol in the choroid and in the individual layers of the rabbit retina. J Biol Chem. 1986;261:4046-4051.

11. Margolis R, Spaide RF. A pilot study of enhanced depth imaging optical coherence tomography of the choroid in normal eyes. Am J Ophthalmol. 2009;147:811-815.

12. Yeoh J, Rahman W, Chen F, Hooper C, Patel P, Tufail A, et al. Choroidal imaging in inherited retinal disease using the technique of enhanced depth imaging optical coherence tomography. Graefes Arch Clin Exp Ophthalmol. 2010;248:1719-1728.

13. Nagaoka T, Kitaya N, Sugawara R, Yokota H, Mori F, Hikichi $\mathrm{T}$, et al. Alteration of choroidal circulation in the foveal region in patients with type 2 diabetes. $\mathrm{Br} \mathrm{J}$ Ophthalmol. 2004;88:1060-1063.

14. Schocket LS, Brucker AJ, Niknam RM, Grunwald JE, Du Pont J, Brucker AJ. Foveolar choroidal hemodynamics in proliferative diabetic retinopathy. Int Ophthalmol. 2004;25:89-94.

15. Margolis R, Spaide RF. A pilot study of enhanced depth imaging optical coherence tomography of the choroid in normal eyes. Am J Ophthalmol 2009;147:811-815.

16. Fujiwara T, Imamura $Y$, Margolis R, Slakter JS, Spaide RF. Enhanced depth imaging optical coherence tomography of the choroid in highly myopic eyes. Am J Ophthalmol. 2009;148:445-450.

17. El Ghonemy K, Rajab GZ, Ibrahim AM, Gohar IM. Comparison between choroidal thickness in patients with diabetic retinopathy and normal individuals using enhanced depth imaging spectral- domain optical coherence tomography. Delta J Ophthalmol. 2018;19:53-57

18. Wang W, Liu S, Qiu Z. Choroidal thickness in diabetes and diabetic retinopathy: a swept source OCT study. Invest Ophthalmol Vis Sci. 2020;61(4):29.
19. Lains I, Talcott KE, Santos AR. Choroidal thickness in diabetic retinopathy assessed with swept-source optical coherence tomography. Retina. 2018;38:173-182.

20. Horvath H, Kovacs I, Sandor GL. Choroidal thickness changes in non-treated eyes of patients with diabetes: Swept-source optical coherence tomography study. Acta Diabetol. 2018;55:927-934.

21. Ambiya V, Kumar A, Baranwal VK. Change in subfoveal choroidal thickness in diabetes and in various grades of diabetic retinopathy. Int J Retina Vitreous. 2018;4:34.

22. Tavares FJ, Vicente A, Proenca R. Choroidal thickness in diabetic patients without diabetic retinopathy. Retina. 2018;38:795-804

23. $\mathrm{Xu} \mathrm{J}, \mathrm{Xu} \mathrm{L}, \mathrm{Du}$ KF. Subfoveal choroidal thickness in diabetes and diabetic retinopathy. Ophthalmology. 2013;120:2023-2028

24. Wang JC, Lains I, Providencia J. Diabetic choroidopathy: choroidal vascular density and volume in diabetic retinopathy with swept-source optical coherence tomography. Am J Ophthalmol. 2017;184:75-83.

25. Zhang X, Ma J, Wang Y. Elevated serum IGF-1 level enhances retinal and choroidal thickness in untreated acromegaly patients. Endocrine. 2018;59:634-642.

26. Nomura Y, Takahashi H, Fujino Y, Kawashima H, Yanagi Y. Association between aqueous humor cxc motif chemokine ligand 13 levels and subfoveal choroidal thickness in normal older subjects. Retina. 2016;36:192-198.

27. Yokouchi H, Baba T, Misawa S. Correlation between serum level of vascular endothelial growth factor and subfoveal choroidal thickness in patients with POEMS syndrome. Graefes Arch Clin Exp Ophthalmol. 2015;253:1641-1646.

28. Querques G, Lattanzio R, Querques L, Enhanced depth imaging optical coherence tomography in type 2 diabetes. Invest Ophthalmol Vis Sci. 2012;53:6017-6024.

\section{Authors' Contributions:}

HH: Conceived the study and manage data collection.

AC: Study was done under her supervision.

MAT: Contributed in acquisition of data, critical review, and is responsible for integrity of the study, final approval of manuscript.

HNN: Contributed in study design and drafting the article.
Authors:

1. Hafsa Hassan, MBBS, FCPS

2. Alyscia Cheema, MBBS, FCPS, FRCS.

Professor of Ophthalmology

3. Muhammad Ali Tahir, MBBS, FCPS (Ophthalmology), FCPS (Vitreoretina)

Consultant Retinal Surgeon

4. Hina Nasreen Nawaz, MBBS, MRCS. TRMO,

1-4: Jinnah Post Graduate Medical Centre, Karachi, Pakistan. 